\title{
Effects of momentum-dependent nuclear potential on two-nucleon correlation functions and light cluster production in intermediate energy heavy-ion collisions
}

\author{
Lie-Wen Chen, ${ }^{1, *}$ Che Ming Ko, ${ }^{1}$ and Bao-An $\mathrm{Li}^{2}$ \\ ${ }^{1}$ Cyclotron Institute and Physics Department, Texas A\&M University, College Station, Texas 77843-3366, USA \\ ${ }^{2}$ Department of Chemistry and Physics, P.O. Box 419, Arkansas State University, State University, Arkansas 72467-0419, USA
}

(Received 16 March 2004; published 14 May 2004)

\begin{abstract}
Using an isospin- and momentum-dependent transport model, we study the effects due to the momentum dependence of isoscalar nuclear potential as well as that of symmetry potential on two-nucleon correlation functions and light cluster production in intermediate energy heavy-ion collisions induced by neutron-rich nuclei. It is found that both observables are affected significantly by the momentum dependence of nuclear potential, leading to a reduction of their sensitivity to the stiffness of nuclear symmetry energy. However, the $t /{ }^{3} \mathrm{He}$ ratio remains a sensitive probe of the density dependence of nuclear symmetry energy.
\end{abstract}

DOI: 10.1103/PhysRevC.69.054606

PACS number(s): 25.70.Pq., 21.30.Fe., 24.10.Lx

\section{INTRODUCTION}

The equation of state (EOS) of an asymmetric nuclear matter with unequal numbers of protons and neutrons depends crucially on the nuclear symmetry energy. Although the nuclear symmetry energy at normal nuclear matter density is known to be around $30 \mathrm{MeV}$ from the empirical liquid-drop mass formula [1,2], its values at other densities are poorly known. Studies based on various theoretical models also give widely different predictions [3]. Lack of this knowledge has hampered our understanding of both the structure of radioactive nuclei [4-7] and many important issues in nuclear astrophysics [8-10], such as the nucleosynthesis during the pre-supernova evolution of massive stars and the properties of neutron stars. However, recent advance in radioactive nuclear beam facilities provides a unique opportunity to study the density dependence of nuclear symmetry energy [3,11-14]. Theoretical studies have already shown that in heavy-ion collisions induced by neutron-rich nuclei, the effect of nuclear symmetry energy can be studied via the ratio of preequilibrium neutrons and protons [15], the isospin fractionation [16-20], the isoscaling in multifragmentation [21], the proton differential elliptic flow [22], the neutronproton differential transverse flow [23,24], the $\pi^{-}$to $\pi^{+}$ratio [25], and isospin diffusion in heavy-ion collisions [26,27]. Also, it was found that the correlation function between nucleon pairs with high total momentum and the isobaric ratio $t /{ }^{3} \mathrm{He}$ in heavy-ion collisions induced by neutron-rich nuclei are sensitive to the density dependence of nuclear symmetry energy [28-31], implying that the space-time properties of neutron and proton emission sources are affected by the nuclear symmetry energy. In these studies, the momentum dependence of nuclear mean-field potential, especially its isovector part (the symmetry potential), was not taken into account. The momentum dependence of nuclear isoscalar potential is well known, and its effect in heavy-ion collisions is large [32]. As shown in Refs. [33-36], the ex-

\footnotetext{
*On leave from Department of Physics, Shanghai Jiao Tong University, Shanghai 200030, China.
}

perimental data on the nucleon directed flow in intermediate energy heavy-ion collisions, which were previously explained by a stiff nuclear equation of state with compressibility of $380 \mathrm{MeV}$ when the momentum dependence of nuclear isoscalar potential was not taken into account, are actually consistent with a soft nuclear equation of state with compressibility of $200 \mathrm{MeV}$ after including the momentum dependence. The momentum dependence of nuclear isoscalar potential was also found to affect the space-time properties of nucleon emission source [37]. The momentum dependence of nuclear symmetry potential is, on the other hand, poorly known. Its effect on some of the isospin observables mentioned above has only recently been studied [38-40] and was found to be significant. In this paper, we shall study how the momentum dependence of both isoscalar nuclear potential and nuclear symmetry potential affect two-nucleon correlation functions and light cluster production in intermediate energy heavy-ion collisions induced by neutron-rich nuclei. Our results show that the momentum dependence of nuclear potential reduces the sensitivity of two-nucleon correlation functions and the light cluster yield on the stiffness of nuclear symmetry energy. However, the $t /{ }^{3} \mathrm{He}$ ratio is still found to depend on the stiffness of nuclear symmetry energy even with the inclusion of its momentum dependence.

This paper is organized as follows. In Sec. II, we discuss the momentum dependence of nuclear isoscalar potential and nuclear symmetry potential. In Sec. III, we present results from the isospin- and momentum-dependent BoltzmannUehling-Uhlenbeck (IBUU) transport model on two-nucleon correlation functions in heavy-ion collisions induced by neutron-rich nuclei at intermediate energies. Results on light cluster production and the $t /{ }^{3} \mathrm{He}$ ratio based on the nucleon coalescence model are given in Sec. IV. Finally, we conclude with a summary in Sec. V.

\section{MOMENTUM DEPENDENCE OF NUCLEAR MEAN-FIELD POTENTIAL}

The energy per nucleon in an asymmetric nuclear matter is usually expressed as 


$$
E(\rho, \delta)=E(\rho, \delta=0)+E_{\mathrm{sym}}(\rho) \delta^{2}+O\left(\delta^{4}\right),
$$

where $\rho=\rho_{n}+\rho_{p}$ is the baryon density with $\rho_{n}$ and $\rho_{p}$ denoting the neutron and proton densities, respectively; $\delta=\left(\rho_{n}\right.$ $\left.-\rho_{p}\right) /\left(\rho_{p}+\rho_{n}\right)$ is the isospin asymmetry; and $E(\rho, \delta=0)$ is the energy per particle in a symmetric nuclear matter, while $E_{\text {sym }}(\rho)$ is the nuclear symmetry energy. Studies based on various many-body theories using nonlocal interactions have shown that the momentum dependence of nuclear single-particle potential is different for neutrons and protons in asymmetric nuclear matter, see, e.g., Ref. [9] for a review. Using the Gogny effective interaction (MDI), the nucleon single-particle potential was recently determined in the mean-field approximation by fitting the saturation properties of nuclear matter at zero temperature with compressibility $K_{0}=211 \mathrm{MeV}$ and symmetry energy $30 \mathrm{MeV}$ [41]. The resulting potential $U(\rho, \delta, \mathbf{p}, \tau)$ for a nucleon with isospin $\tau(1 / 2$ for protons and $-1 / 2$ for neutrons) and momentum $\mathbf{p}$ in an asymmetric nuclear matter with isospin asymmetry $\delta$ and density $\rho$, can be parametrized as

$$
\begin{aligned}
U(\rho, \delta, \mathbf{p}, \tau)= & A_{u} \frac{\rho_{\tau^{\prime}}}{\rho_{0}}+A_{l} \frac{\rho_{\tau}}{\rho_{0}}+B\left(\frac{\rho}{\rho_{0}}\right)^{\sigma}\left(1-x \delta^{2}\right) \\
& -8 \tau x \frac{B}{\sigma+1} \frac{\rho^{\sigma-1}}{\rho_{0}^{\sigma}} \delta \rho_{\tau^{\prime}} \\
& +\frac{2 C_{\tau, \tau}}{\rho_{0}} \int d^{3} \mathbf{p}^{\prime} \frac{f_{\tau}\left(\mathbf{r}, \mathbf{p}^{\prime}\right)}{1+\left(\mathbf{p}-\mathbf{p}^{\prime}\right)^{2} / \Lambda^{2}} \\
& +\frac{2 C_{\tau, \tau^{\prime}}}{\rho_{0}} \int d^{3} \mathbf{p}^{\prime} \frac{f_{\tau^{\prime}}\left(\mathbf{r}, \mathbf{p}^{\prime}\right)}{1+\left(\mathbf{p}-\mathbf{p}^{\prime}\right)^{2} / \Lambda^{2}},
\end{aligned}
$$

where $\tau \neq \tau^{\prime}$ and $f_{\tau}(\mathbf{r}, \mathbf{p})$ denotes the phase-space distribution function at coordinate $\mathbf{r}$ and momentum $\mathbf{p}$. The parameter $x$ in Eq. (2) is introduced to reflect the uncertainty of our knowledge on the density dependence of nuclear symmetry energy, and it has a value of 1 in the Gogny interaction [41]. For the momentum-independent part of the potential, given by the first two lines of Eq. (2), the value for $\sigma$ is $3 / 4$ and for $B$ is $106.35 \mathrm{MeV}$, while those for $A_{l}$ and $A_{u}$ depend on the value of $x$. For $x=0$, we have $A_{l}=-120.57 \mathrm{MeV}$ and $A_{u}$ $=-95.98 \mathrm{MeV}$. For other values of $x$, they are

$$
A_{l}(x)=A_{l}(0)+\frac{2 B}{\sigma+1} x, \quad A_{u}(x)=A_{u}(0)-\frac{2 B}{\sigma+1} x,
$$

with $A_{l}(0)$ and $A_{u}(0)$ denoting the values for $x=0$.

The terms with parameters $C_{\tau, \tau^{\prime}}=-103.4 \mathrm{MeV}$ and $C_{\tau, \tau}$ $=-11.7 \mathrm{MeV}$ with $\Lambda=1.0 p_{F}^{0}$, where $p_{F}^{0}$ denotes the Fermi momentum at normal nuclear matter density, in the second line of Eq. (2) describes the momentum dependence of not only the nuclear isoscalar potential but also the nuclear symmetry potential as a nucleon with isospin $\tau$ interacts differently with unlike and like nucleons in the background fields.

With different $x$ values, one can obtain different density dependence for the nuclear symmetry energy without changing other properties of the asymmetric nuclear matter. As seen in Fig. 1, where the density dependence of nuclear symmetry energy from the MDI interaction with different $x$ val-

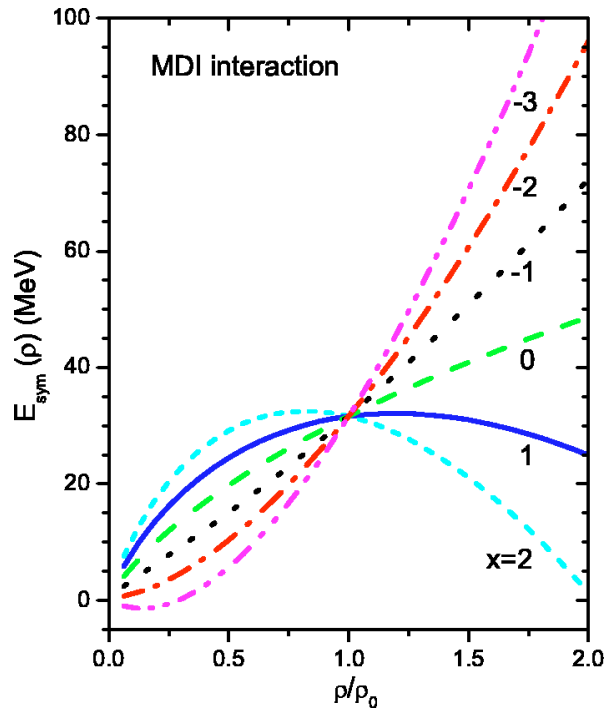

FIG. 1. (Color online) Density dependence of nuclear symmetry energy from the MDI interaction with different $x$ values.

ues are shown, the symmetry energy becomes stiffer with decreasing $x$ but its value at normal nuclear density remains the same. To explore the large range of $E_{\mathrm{sym}}(\rho)$ predicted by various many-body theories [5,42-44], we consider in the present study two values of $x=1$ and $x=-2$. Since the nuclear symmetry energy given by $x=1$ has a weaker dependence on density than the one given by $x=-2$, we call it a soft symmetry energy while the one corresponding to $x=-2$ is called a hard symmetry energy. We note that the soft symmetry energy with $x=1$ is the same as that given by the default Gogny interaction.

The difference in the density dependence of soft and stiff symmetry energies is due to the different density dependence in the momentum-independent part of the potential from the MDI interaction as shown in Fig. 2, where we give the den-

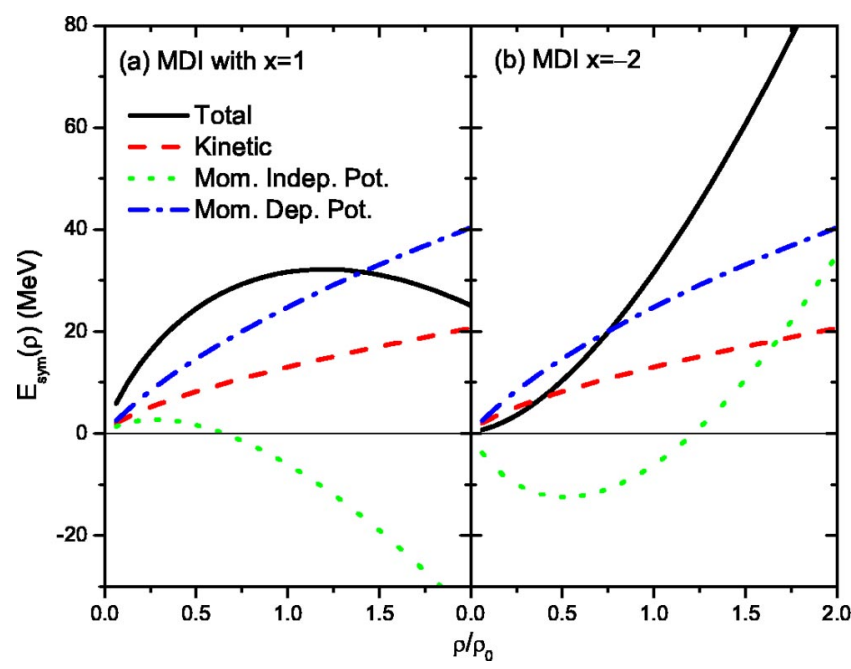

FIG. 2. (Color online) Density dependence of total symmetry energy together with contributions from the kinetic energy part as well as the momentum-independent and momentum-dependent potential energy parts from the MDI interaction with (a) $x=1$ and (b) $x=-2$. 


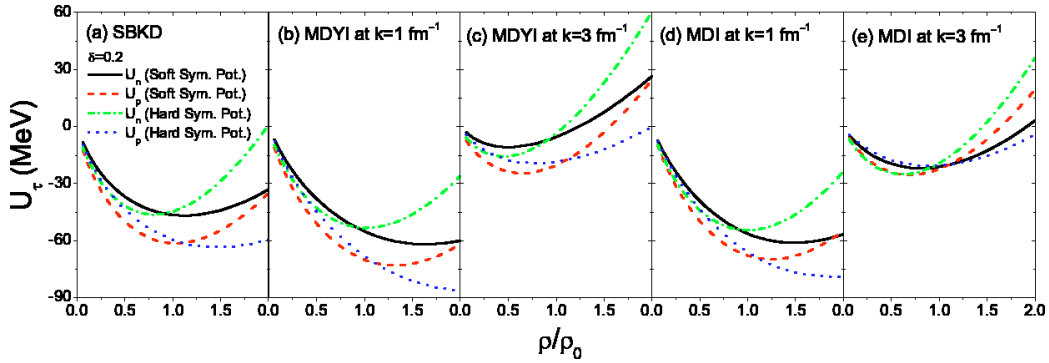

FIG. 3. (Color online) Density dependence of neutron and proton single-particle potentials at different momenta in asymmetric nuclear matter with isospin asymmetry $\delta=0.2$ for different nucleon effective interactions. sity dependence of total symmetry energy for these two cases together with that due to contributions from the kinetic energy part as well as the momentum-independent and momentum-dependent potential energy parts. The total potential energy contribution to the symmetry energy shown in Fig. 2 can be parametrized as

$$
E_{\mathrm{sym}}^{\mathrm{pot}}(\rho)=3.08+39.6 u-29.2 u^{2}+5.68 u^{3}-0.523 u^{4}(\mathrm{MeV}),
$$

for the soft symmetry energy, i.e., the MDI with $x=1$ and

$$
E_{\mathrm{sym}}^{\mathrm{pot}}(\rho)=-1.83-5.45 u+30.34 u^{2}-5.04 u^{3}+0.45 u^{4}(\mathrm{MeV})
$$

for the hard symmetry energy, i.e., the MDI with $x=-2$. In the above, $u \equiv \rho / \rho_{0}$ is the reduced nucleon density.

To study the effects due to the momentum dependence of nuclear symmetry energy, we also consider a nucleon potential $U_{\text {noms }}(\rho, \delta, \mathbf{p}, \tau) \equiv U_{0}(\rho, \mathbf{p})+U_{\text {sym }}(\rho, \delta, \tau)$ with the isoscalar part taken from the original MDYI interaction [34], i.e.,

$$
\begin{aligned}
U_{0}(\rho, \mathbf{p})= & -110.44 u+140.9 u^{1.24} \\
& -\frac{130}{\rho_{0}} \int d^{3} \mathbf{p}^{\prime} \frac{f\left(\mathbf{r}, \mathbf{p}^{\prime}\right)}{1+\left(\mathbf{p}-\mathbf{p}^{\prime}\right)^{2} /\left(1.58 p_{F}^{0}\right)^{2}},
\end{aligned}
$$

which has a compressibility $K_{0}=215 \mathrm{MeV}$ and is almost the same as the momentum-dependent isoscalar potential given by the MDI interaction, i.e., Eq. (2). For the momentum-independent symmetry potential $U_{\text {sym }}(\rho, \delta, \tau)$, it is obtained from $U_{\text {sym }}(\rho, \delta, \tau)=\partial W_{\text {sym }} / \partial \rho_{\tau}$ using the isospin-dependent part of the potential energy density $W_{\mathrm{sym}}=E_{\mathrm{sym}}^{\mathrm{pot}}(\rho) \cdot \rho \cdot \delta^{2}$ where $E_{\mathrm{sym}}^{\mathrm{pot}}(\rho)$ is given by Eqs. (4) and (5) from the MDI interaction.
To study the effects due to the momentum dependence of isoscalar nuclear potential, we also consider the usual momentum-independent soft nuclear isoscalar potential with $K_{0}=200 \mathrm{MeV}$ (SBKD), first introduced by Bertsch, Kruse and Das Gupta [45], i.e.,

$$
U(\rho)=-356 u+303 u^{7 / 6} .
$$

The density dependence of neutron and proton single-particle potentials at isospin asymmetry $\delta=0.2$ and at momenta $k$ $=1 \mathrm{fm}^{-1}$ and $k=3 \mathrm{fm}^{-1}$ are shown in Fig. 3 for the SBKD, MDYI, and MDI interactions. It is seen that neutrons generally have a stronger repulsive potential than protons. Their difference further depends on the nuclear symmetry energy, with the soft one giving a larger difference at low densities while the hard one giving a larger difference at high densities. Also, with momentum-dependent MDYI and MDI interactions, the nuclear potential is more repulsive for high momentum nucleons. Moreover, the momentum dependence of nuclear symmetry potential from the MDI interaction generally reduces the difference between the neutron and proton potentials, especially at high momenta.

To see more clearly the momentum dependence of nucleon mean-field potential, we show in Fig. 4 the momentum dependence of neutron and proton single-particle potentials in asymmetric nuclear matter with isospin asymmetry $\delta=0.2$ and at different densities for different nucleon effective interactions. Although the MDI interaction is seen to give a similar momentum dependence in the nucleon potential as the MDYI interaction, the difference between the neutron and proton potentials, is reduced, especially at higher momenta, due to its momentum dependence in the nuclear symmetry potential. Therefore, the MDI interaction gives a weaker symmetry potential than that from the MDYI interaction.

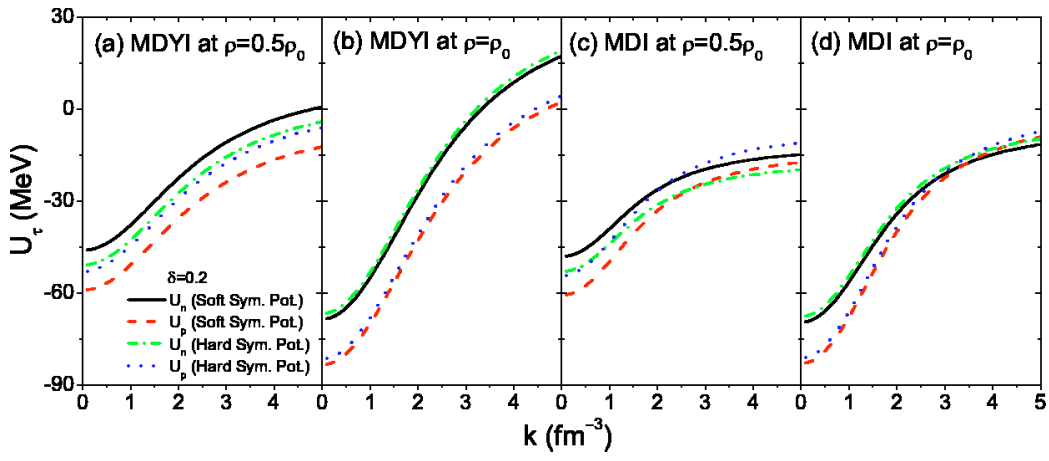

054606-3
FIG. 4. (Color online) Momentum dependence of neutron and proton single-particle potentials in asymmetric nuclear matter with isospin asymmetry $\delta=0.2$ and at different densities for different nucleon effective interactions. 


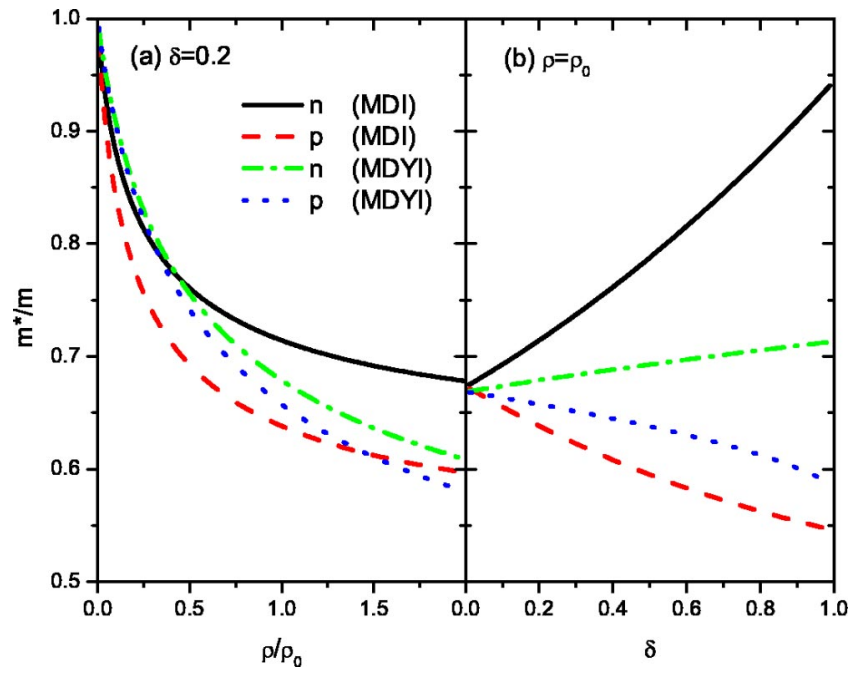

FIG. 5. (Color online) (a) Density and (b) isospin asymmetry dependence of nucleon effective mass in asymmetric nuclear matter with isospin asymmetry $\delta=0.2$ and at normal nuclear matter density for the MDYI and MDI interactions.

The nucleon effective mass is an important quantity to characterize the momentum dependence of nuclear meanfield potential. From the single-particle potential, we can obtain the nucleon effective mass from

$$
\frac{m_{\tau}^{*}}{m_{\tau}}=\left(1+\frac{m_{\tau}}{p} \frac{d U}{d p}\right)_{p=p_{f}(\tau)}^{-1}
$$

In Fig. 5, we show both the density (a) and isospin asymmetry (b) dependence of nucleon effective mass in asymmetric nuclear matter with isospin asymmetry $\delta=0.2$ and at normal nuclear matter density for the MDYI and MDI interactions. It is seen that a neutron has a larger effective mass than a proton in neutron-rich nuclear matter and both depends strongly on the isospin asymmetry of nuclear matter. The small difference between the neutron and proton effective masses for the MDYI interaction is due to the different neutron and proton Fermi energies. In addition, the MDYI interaction shows a slightly stronger density dependence for the nucleon effective mass than the MDI interaction.

Above discussions indicate that the nucleon symmetry potential is reduced after including its momentum dependence and the effect gets larger with increasing nucleon momentum. The difference between symmetry potentials with and without momentum dependence is thus larger for nucleons with higher momenta. In the following, the three potentials obtained with the SBKD, MDYI, and MDI interactions will be used together with the isospin-dependent experimental $N N$ cross sections in the IBUU model $[15,38,39]$ to study the effects due to the momentum dependence of isoscalar and isovector (symmetry) potentials on two-nucleon correlation functions and light cluster production in intermediate energy heavy-ion collisions induced by neutron-rich nuclei. In previous studies $[12,15,28]$, it has been shown that these observables are sensitive to the symmetry potential but not to the isoscalar potential and the in-medium nucleon-nucleon cross sections.

\section{TWO-NUCLEON CORRELATION FUNCTIONS}

The space-time properties of nucleon emission source, which are important for understanding the reaction dynamics of heavy-ion collisions, can be extracted from two-particle correlation functions; see, e.g., Refs. [46-49] for reviews. In most studies, only the two-proton correlation function is studied [50-56]. Recently, data on two-neutron and neutronproton correlation functions have also become available. The neutron-proton correlation function is especially useful as it is free of correlations due to wave-function antisymmetrization and Coulomb interactions. Indeed, Ghetti et al. have deduced from measured neutron-proton correlation function the emission sequence of neutrons and protons in intermediate energy heavy-ion collisions [57-59] and have also studied the isospin effects on two-nucleon correlation functions [60].

In standard Koonin-Pratt formalism [61-63], the twoparticle correlation function is obtained by convoluting the emission function $g(\mathbf{p}, x)$, i.e., the probability for emitting a particle with momentum $\mathbf{p}$ from the space-time point $x$ $=(\mathbf{r}, t)$, with the relative wave function of the two particles, i.e.,

$$
C(\mathbf{P}, \mathbf{q})=\frac{\int d^{4} x_{1} d^{4} x_{2} g\left(\mathbf{P} / 2, x_{1}\right) g\left(\mathbf{P} / 2, x_{2}\right)|\phi(\mathbf{q}, \mathbf{r})|^{2}}{\int d^{4} x_{1} g\left(\mathbf{P} / 2, x_{1}\right) \int d^{4} x_{2} g\left(\mathbf{P} / 2, x_{2}\right)} .
$$

In the above, $\mathbf{P}\left(=\mathbf{p}_{1}+\mathbf{p}_{2}\right)$ and $\mathbf{q}\left[=\frac{1}{2}\left(\mathbf{p}_{1}-\mathbf{p}_{2}\right)\right]$ are, respectively, the total and relative momenta of the particle pair; and $\phi(\mathbf{q}, \mathbf{r})$ is the relative two-particle wave function with $\mathbf{r}$ being their relative position, i.e., $\mathbf{r}=\left(\mathbf{r}_{2}-\mathbf{r}_{1}\right)-\frac{1}{2}\left(\mathbf{v}_{1}+\mathbf{v}_{2}\right)\left(t_{2}-t_{1}\right)$. This approach has been very useful in studying effects of nuclear equation of state and nucleon-nucleon cross sections on the reaction dynamics of intermediate energy heavy-ion collisions [47]. In the present paper, we use the Koonin-Pratt method to determine the nucleon-nucleon correlation functions in order to study the effect due to the momentum dependence of nuclear mean-field potential and the density dependence of nuclear symmetry energy on the spatial and temporal properties of nucleon emission source in intermediate energy heavy-ion collisions.

As an example, we study here central collisions of ${ }^{52} \mathrm{Ca}$ $+{ }^{48} \mathrm{Ca}$ at $E=80 \mathrm{MeV} /$ nucleon. This particular reaction system with isospin asymmetry $\delta=0.2$ can be studied at the future Rare Isotope Accelerator (RIA). In the present work, nucleons are considered as emitted when their local densities are less than $\rho_{0} / 8$ and subsequent interactions do not cause their recapture into regions of higher density. In Fig. 6, we show the emission rates of protons and neutrons as functions of time for the SBKD, MDYI, and MDI interactions with soft and hard symmetry energies. It is clearly seen that there are two stages of nucleon emissions; an early fast emission and a subsequent slow emission. This is consistent with the long-lived nucleon emission source observed in previous 


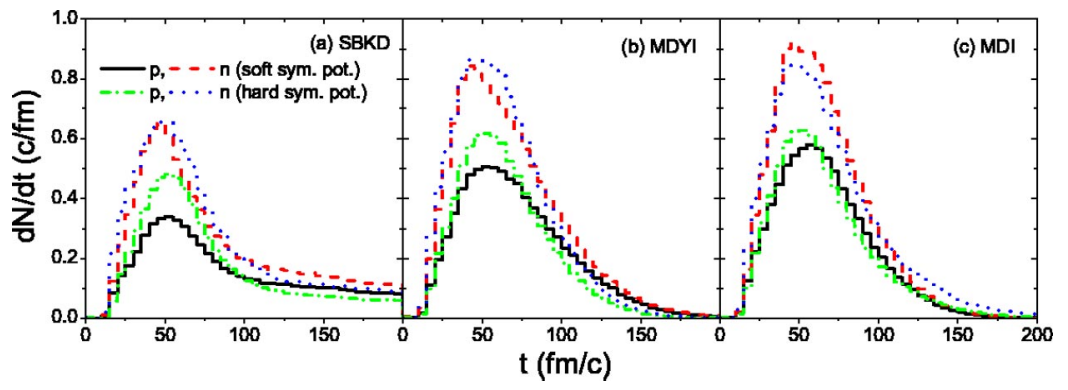

FIG. 6. (Color online) Emission rates of protons and neutrons as functions of time for different nucleon effective interactions.

BUU calculations [54]. For the momentum-independent nuclear potential (SBKD), we find from Fig. 6(a) that the hard symmetry energy enhances the emission of early high momentum protons (dash-dotted line) and neutrons (dotted line) but suppresses late slow emission compared with results from the soft symmetry energy (protons and neutrons are given by solid and dashed lines, respectively). The difference between the emission rates of protons and neutrons is, however, larger for the soft symmetry energy. Figure 6(b) shows results from the MDYI interaction which includes the momentum-dependent isoscalar potential but the momentum-independent symmetry potential. It is seen that the momentum dependence of isoscalar potential enhances significantly the nucleon emission rate due to the more repulsive momentum-dependent nuclear potential at high momenta. As a result, the relative effect due to the symmetry potential is reduced compared with the results shown in Fig. 6(a). In Fig. 6(c), we use the MDI interaction which includes momentum dependence in both isoscalar potential and symmetry potential. The momentum dependence of symmetry potential leads to a slightly faster nucleon emission but the symmetry potential effects are reduced. We note that the fraction of total number of nucleons emitted before $200 \mathrm{fm} / c$ in the IBUU simulations is about $80 \%$ for the SBKD interaction but almost $100 \%$ for the MDYI and MDI interactions. The enhancement of nucleon emissions with momentumdependent nuclear mean-field potential was also observed in previous calculations [37]. It should be noted that the emitted nucleons in the present study are not exactly those observed experimentally since we have not included explicitly the production of clusters and intermediate mass fragments in the IBUU simulations.

Using the program Correlation After Burner [64], which takes into account final-state nucleon-nucleon interactions, we have evaluated two-nucleon correlation functions from the emission function given by the IBUU model. Shown in Fig. 7 are two-nucleon correlation functions gated on the total momentum $P$ of nucleon pairs from central collisions of ${ }^{52} \mathrm{Ca}+{ }^{48} \mathrm{Ca}$ at $E=80 \mathrm{MeV} /$ nucleon by using the SBKD interaction with the soft and hard symmetry potentials. The left and right panels are for $P<300 \mathrm{MeV} / c$ and $P>500 \mathrm{MeV} / c$, respectively. Both neutron-neutron (upper panels) and neutron-proton (lower panels) correlation functions peak at $q \approx 0 \mathrm{MeV} / c$, while the proton-proton correlation function (middle panel) is peaked at about $q$ $=20 \mathrm{MeV} / c$ due to the strong final-state $s$-wave attraction. The latter is suppressed at $q=0$ as a result of Coulomb repulsion and antisymmetrization of the two-proton wave function. These general features are consistent with those ob- served in experimental data from heavy-ion collisions [57].

For nucleon pairs with high total momentum, their correlation function is stronger for the hard symmetry energy than for the soft symmetry energy: about $24 \%$ and $9 \%$ for neutron-proton pairs and neutron-neutron pairs at low relative momentum $q=5 \mathrm{MeV} / c$, respectively, and $21 \%$ for proton-proton pairs at $q=20 \mathrm{MeV} / c$. The neutron-proton correlation function thus exhibits the highest sensitivity to the density dependence in nuclear symmetry energy $E_{\text {sym }}(\rho)$. For nucleon pairs with low total momenta, the symmetry potential effects are weak. These results are again consistent with previous calculations [28,29].

What will happen to the symmetry energy effect on twonucleon correlation functions if we include the momentumdependent isoscalar potential in the IBUU model? This can be seen from Fig. 8, where they are shown for the same central collisions of ${ }^{52} \mathrm{Ca}+{ }^{48} \mathrm{Ca}$ at $E=80 \mathrm{MeV} /$ nucleon by using the MDYI interaction with soft and hard symmetry potentials in the IBUU model. For nucleon pairs with low total momentum, shown in left panels, their correlation functions remain insensitive to the nuclear symmetry energy. For nucleon pairs with high total momentum, shown in right panels, their correlation function is again stronger for the hard

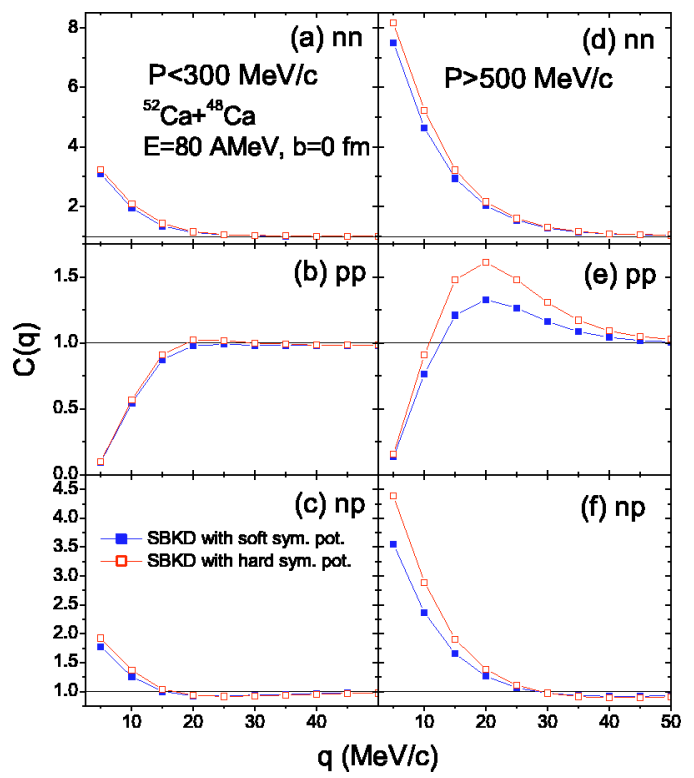

FIG. 7. (Color online) Two-nucleon correlation functions gated on the total momentum $P$ of nucleon pairs using the SBKD interaction with soft (filled squares) or stiff (open squares) symmetry energy. Left panels are for $P<300 \mathrm{MeV} / c$ while right panels are for $P>500 \mathrm{MeV} / c$. 


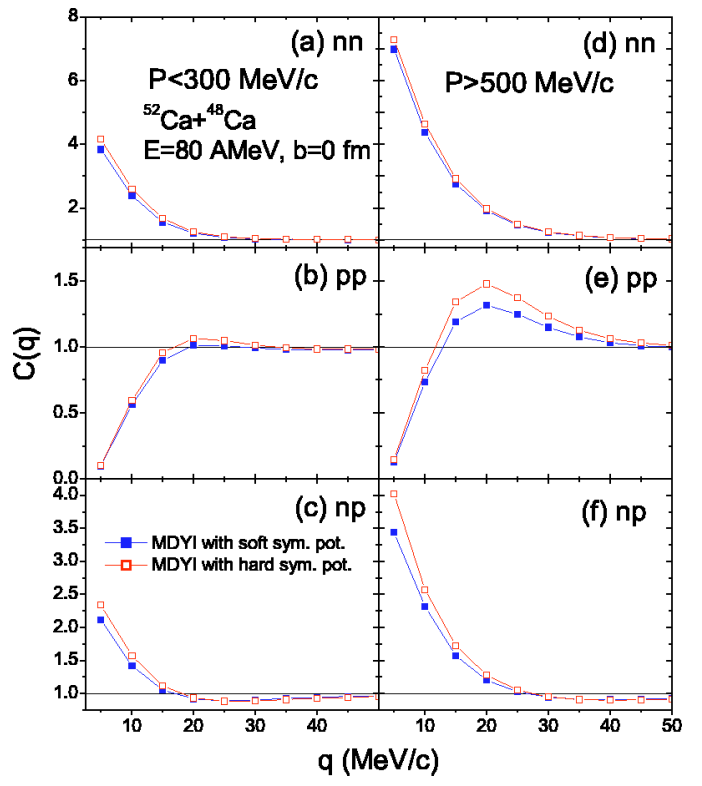

FIG. 8. (Color online) Same as in Fig. 7 but using the MDYI interaction with soft (filled squares) or stiff (open squares) symmetry energy.

symmetry energy than for the soft symmetry energy: about $17 \%$ and $4 \%$ for neutron-proton pairs and neutron-neutron pairs at low relative momentum $q=5 \mathrm{MeV} / c$, respectively, and $12 \%$ for proton-proton pairs at $q=20 \mathrm{MeV} / c$. Compared to the results from the SBKD interaction, the momentum dependence of isoscalar potential thus reduces the symmetry potential effects on two-nucleon correlation functions. This is due to the fact that the repulsive momentum-dependent potential enhances nucleon emissions and thus reduces the density effect on nucleon emissions, leading to a weaker symmetry potential effects on two-nucleon correlation functions.

To see how the momentum dependence of nuclear symmetry energy affects the effect due to its density dependence on two nucleon correlation functions, we show in Fig. 9 the results from the MDI interaction with the soft and hard symmetry potentials for central collisions of ${ }^{52} \mathrm{Ca}+{ }^{48} \mathrm{Ca}$ at $E$ $=80 \mathrm{MeV} /$ nucleon . It is seen that the effect due to the stiffness of nuclear symmetry energy is again small for nucleon pairs with low total momentum as shown in left panels. For nucleon pairs with high total momentum, shown in right panels, their correlation function remains stronger for the hard symmetry energy than for the soft symmetry energy: about $11 \%$ for neutron-proton pairs at low relative momentum $q$ $=5 \mathrm{MeV} / \mathrm{c}$ and $9 \%$ for proton-proton pairs at $q$ $=20 \mathrm{MeV} / c$. The symmetry potential effect on the correlation function of neutron-neutron pairs is, however, very weak. Compared with results from the SBKD and MDYI interactions, the two-nucleon correlation functions from the MDI interaction are thus smaller, mainly due to the very small difference between its neutron and proton potentials, especially for higher momentum nucleons as shown in Fig. 3 and 4 .

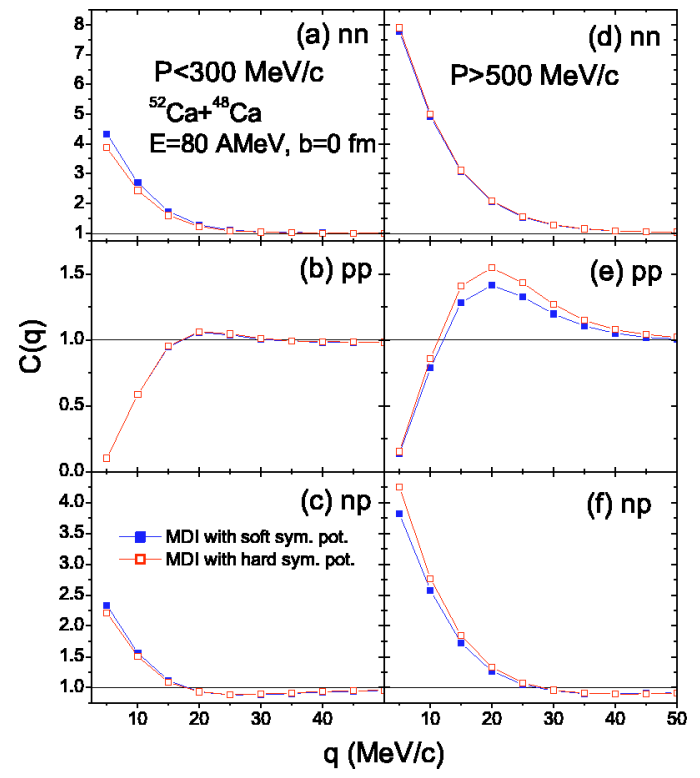

FIG. 9. (Color online) Same as in Fig. 7 but using the MDI interaction with soft (filled squares) or stiff (open squares) symmetry energy.

\section{THE $t /{ }^{3} \mathrm{He}$ RATIO}

Light cluster production has been extensively studied in experiments involving heavy-ion collisions at all energies, e.g., see Ref. [65] for a recent review. A popular model for describing the production of light clusters in these collisions is the coalescence model, e.g., see Ref. [66] for a theoretical review, which has been used at both intermediate [67-69] and high energies [70,71]. In this model, the probability for producing a cluster is determined by the overlap of its Wigner phase-space density with the nucleon phase-space distribution at freeze out. Explicitly, the multiplicity of a $M$-nucleon cluster in a heavy-ion collision is given by [70]

$$
\begin{aligned}
N_{M}= & G \int d \mathbf{r}_{i_{1}} d \mathbf{q}_{i_{1}} \cdots d \mathbf{r}_{i_{M-1}} d \mathbf{q}_{i_{M-1}} \\
& \times\left\langle\sum_{i_{1}>i_{2}>\ldots>i_{M}} \rho_{i}^{W}\left(\mathbf{r}_{i_{1}}, \mathbf{q}_{i_{1}} \cdots \mathbf{r}_{i_{M-1}}, \mathbf{q}_{i_{M-1}}\right)\right\rangle .
\end{aligned}
$$

In the above, $\mathbf{r}_{i_{1}}, \cdots, \mathbf{r}_{i_{M-1}}$ and $\mathbf{q}_{i_{1}}, \cdots, \mathbf{q}_{i_{M-1}}$ are, respectively, the $M-1$ relative coordinates and momenta taken at equal time in the $M$-nucleon rest frame; $\rho_{i}^{W}$ is the Wigner phasespace density of the $M$-nucleon cluster; and $\langle\cdots\rangle$ denotes event averaging. The spin-isospin statistical factor for the cluster is given by $G$, and its value is $3 / 8$ for deuteron and $1 / 3$ for triton or ${ }^{3} \mathrm{He}$, with the latter including the possibility of coalescence of a deuteron with another nucleon to form a triton or ${ }^{3} \mathrm{He}$ [72]. Details about such calculation can be found in Ref. [31].

Shown in Fig. 10 are the kinetic energy spectra in the center-of-mass system for deuterons (first row), tritons (second row), and ${ }^{3} \mathrm{He}$ 's (third row) from central collisions of ${ }^{52} \mathrm{Ca}+{ }^{48} \mathrm{Ca}$ at $E=80 \mathrm{MeV} /$ nucleon by using the SBKD (first column), MDYI (second column) and MDI (third column) interactions with the soft (solid squares) and stiff (open 


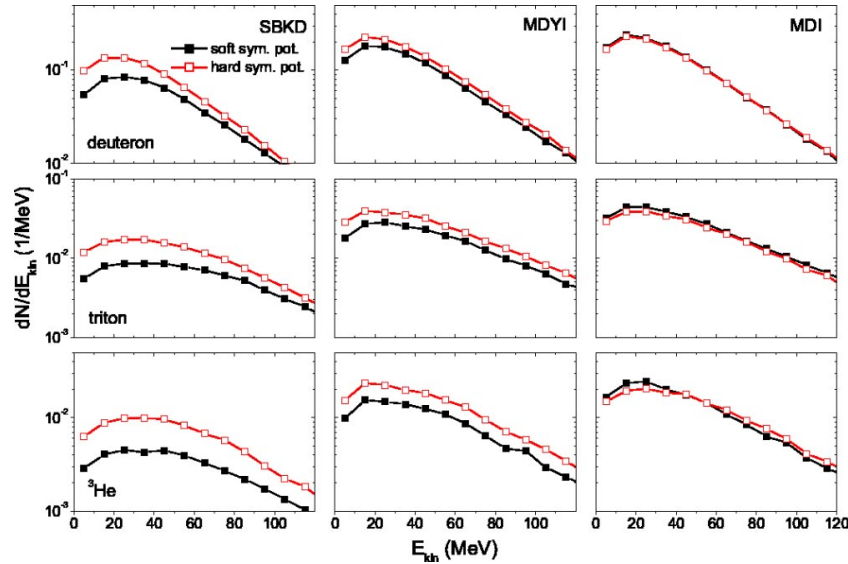

FIG. 10. (Color online) Kinetic energy spectra in the center-ofmass system for deuteron (first row), triton (second row), and ${ }^{3} \mathrm{He}$ (third row) from central collisions of ${ }^{52} \mathrm{Ca}+{ }^{48} \mathrm{Ca}$ at $E$ $=80 \mathrm{MeV} /$ nucleon by using the SBKD (first column), MDYI (second column) and MDI (third column) interactions with the soft (solid squares) or stiff (open squares) symmetry energy.

squares) symmetry energies. For the SBKD interaction, the yields of these light clusters are very sensitive to the density dependence of nuclear symmetry energy, which is consistent with previous studies [30,31]. Including momentum dependence in the isoscalar potential by using the MDYI interaction in the IBUU model reduces slightly the symmetry potential effect, although the yields of light clusters are increased significantly as a result of enhanced nucleon emissions from the momentum-dependent nuclear potential as shown in Fig. 6. If we further include the momentum dependence in symmetry potential by using the MDI interaction, the final yields of light clusters become even less sensitive to the density dependence of symmetry potential than those from the MDYI interaction. This is due to the very small difference between the neutron and proton potentials and the momentum dependence of their symmetry potentials in the MDI interaction as discussed above. It should be noted that the coalescence model is expected to be a reasonable model for describing the production of light clusters with large kinetic energies as they are not contaminated by contributions from decays of heavy fragments, which are mainly of low kinetic energies and may not be negligible in intermediate energy heavy-ion collisions.
The isobaric yield ratio $t /{ }^{3} \mathrm{He}$ is less model-dependent and also less affected by other effects, such as the feedback from heavy fragment evaporation and the feed-down from produced excited triton and ${ }^{3} \mathrm{He}$ states. In Fig. 11, we show the $t /{ }^{3} \mathrm{He}$ ratio with statistical errors as a function of cluster kinetic energy in the center-of-mass system for the SBKD, MDYI and MDI interactions with the soft (solid squares) and stiff (open squares) symmetry energies. For all nuclear potentials, it is seen that the ratio $t /{ }^{3} \mathrm{He}$ obtained with different symmetry energies exhibits very different energy dependence. While the $t /{ }^{3} \mathrm{He}$ ratio increases with kinetic energy for the soft symmetry energy, it decreases and/or increase weakly with kinetic energy for the stiff symmetry energy. For both soft and stiff symmetry energies, the ratio $t /{ }^{3} \mathrm{He}$ is larger than the neutron to proton ratio of the whole reaction system, i.e., $N / Z=1.5$. This is in agreement with results from both experiments and the statistical model simulations for other reaction systems and incident energies [73-77]. It is interesting to note that although the yield of light clusters is not so sensitive to the density dependence of symmetry potential for the MDI interaction, the $t /{ }^{3} \mathrm{He}$ ratio shows very different energy dependence for the soft and hard symmetry potentials. This is related to the different momentum dependence of symmetry potential in the MDI interaction, especially at low densities, as shown in Fig. 4.

\section{SUMMARY}

Through studying two-nucleon correlation functions and light cluster production using an isospin- and momentumdependent transport model, we have investigated the effects due to the momentum dependence of isoscalar nuclear potential and also the symmetry potential on the space-time properties of nucleon emission source in heavy-ion collisions induced by neutron-rich nuclei at intermediate energies. It is found that the momentum dependence of both isoscalar nuclear potential and the symmetry potential influences significantly the space-time properties of nucleon emission source, leading thus to appreciable effects on two-nucleon correlation functions and light cluster production in these collisions. Specifically, the momentum dependence of nuclear potential reduces the sensitivity of two-nucleon correlation functions and the light cluster yield on the stiffness

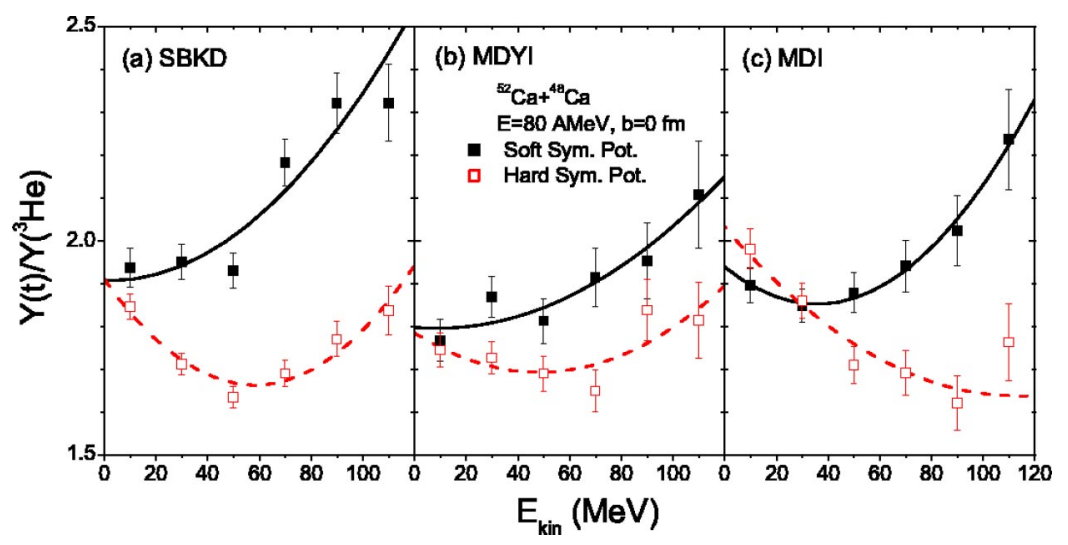

FIG. 11. (Color online) The $t /{ }^{3} \mathrm{He}$ ratio as a function of cluster kinetic energy in the center-ofmass system for different interactions (a) SBKD, (b) MDYI, and (c) MDI with the soft (solid squares) and stiff (open squares) symmetry energies. The lines are drawn to guide the eyes. 
of nuclear symmetry energy. However, the $t /{ }^{3} \mathrm{He}$ ratio is still found to be sensitive to the stiffness of symmetry energy after including the momentum dependence of nuclear potential. The study of these observables in intermediate energy heavy-ion collisions thus offers the possibility to probe both the momentum dependence of nuclear symmetry potential and the density dependence of nuclear symmetry energy.

\section{ACKNOWLEDGMENTS}

This paper was based on work supported by the U.S. National Science Foundation under Grant Nos. PHY-0098805, PHY-0088934, and PHY-0243571 as well as the Welch Foundation under Grant No. A-1358. L.W.C. was also supported by the National Natural Science Foundation of China under Grant No. 10105008.
[1] W. D. Myers and W. J. Swiatecki, Nucl. Phys. 81, 1 (1966).

[2] K. Pomorski and J. Dudek, Phys. Rev. C 67, 044316 (2003).

[3] Isospin Physics in Heavy-Ion Collisions at Intermediate Energies, edited by Bao-An Li and W. Udo Schröder (Nova Science, New York, 2001).

[4] K. Oyamatsu, I. Tanihata, Y. Sugahara, K. Sumiyoshi, and H. Toki, Nucl. Phys. A634, 3 (1998).

[5] B. A. Brown, Phys. Rev. Lett. 85, 5296 (2000).

[6] C. J. Horowitz and J. Piekarewicz, Phys. Rev. Lett. 86, 5647 (2001); Phys. Rev. C 63, 025501 (2001).

[7] R. J. Furnstahl, Nucl. Phys. A706, 85 (2002).

[8] H. A. Bethe, Rev. Mod. Phys. 62, 801 (1990).

[9] I. Bombaci, in [3], p. 35.

[10] J. M. Lattimer and M. Prakash, Astrophys. J. 550, 426 (2001).

[11] Radioactive Nuclear Beams, edited by I. Tanihata, Nucl. Phys. A693, 1 (2001).

[12] B. A. Li, C. M. Ko, and W. Bauer, topical review, Int. J. Mod. Phys. E 7, 147 (1998).

[13] B. A. Li, Phys. Rev. C 69, 034614 (2004).

[14] B. A. Li, Nucl. Phys. A (to be published), nucl-th/0312026.

[15] B. A. Li, C. M. Ko, and Z. Z. Ren, Phys. Rev. Lett. 78, 1644 (1997).

[16] B. A. Li and C. M. Ko, Nucl. Phys. A618, 498 (1997).

[17] V. Baran, M. Colonna, M. Di Toro, and A. B. Larionov, Nucl. Phys. A632, 287 (1998).

[18] H. S. Xu et al., Phys. Rev. Lett. 85, 716 (2000).

[19] W. P. Tan et al., Phys. Rev. C 64, 051901(R) (2001).

[20] V. Baran, M. Colonna, M. Di Toro, V. Greco, M. ZielinskaPfabé, and H. H. Wolter, Nucl. Phys. A703, 603 (2002).

[21] M. B. Tsang et al., Phys. Rev. Lett. 86, 5023 (2001).

[22] B. A. Li, A. T. Sustich, and B. Zhang, Phys. Rev. C 64, 054604 (2001).

[23] B. A. Li, Phys. Rev. Lett. 85, 4221 (2000).

[24] V. Greco, V. Baran, M. Colonna, M. Di Toro, T. Gaitanos, and H. H. Wolter, Phys. Lett. B 562, 215 (2003).

[25] B. A. Li, Phys. Rev. Lett. 88, 192701 (2002); Nucl. Phys. A708, 365 (2002).

[26] L. Shi and P. Danielewicz, Phys. Rev. C 68, 064604 (2003).

[27] M. B. Tsang et al., Phys. Rev. Lett. 92, 062701 (2004).

[28] L. W. Chen, V. Greco, C. M. Ko, and B. A. Li, Phys. Rev. Lett. 90, 162701 (2003).

[29] L. W. Chen, V. Greco, C. M. Ko, and B. A. Li, Phys. Rev. C 68, 014605 (2003).

[30] L. W. Chen, C. M. Ko, and B. A. Li, Phys. Rev. C 68, 017601 (2003)

[31] L. W. Chen, C. M. Ko, and B. A. Li, Nucl. Phys. A729, 809 (2003).

[32] P. Danielewicz, R. Lacey, and W. G. Lynch, Science 298,
1592 (2002).

[33] C. Gale et al., Phys. Rev. C 35, 1666 (1987).

[34] G. M. Welke et al., Phys. Rev. C 38, 2101 (1988); C. Gale et al., ibid. 41, 1545 (1990).

[35] Q. Pan and P. Danielewicz, Phys. Rev. Lett. 70, 2062 (1993).

[36] J. Zhang et al., Phys. Rev. C 50, 1617 (1994).

[37] V. Greco, A. Guarnera, M. Colonna, and M. Di Toro, Phys. Rev. C 59, 810 (1999).

[38] B. A. Li, C. B. Das, S. Das Gupta, and C. Gale, Phys. Rev. C 69, 011603(R) (2004).

[39] B. A. Li, C. B. Das, S. Das Gupta, and C. Gale, Nucl. Phys. A735, 563 (2004).

[40] J. Rizzo, M. Colonna, M. Di Toro, and V. Greco, Nucl. Phys. A732, 202 (2004).

[41] C. B. Das et al., Phys. Rev. C 67, 034611 (2003).

[42] R. B. Wiringa et al., Phys. Rev. C 38, 1010 (1988).

[43] I. Bombaci et al., Phys. Rev. C 44, 1892 (1991).

[44] V. Greco, M. Colonna, M. Di Toro, G. Fabbri, and F. Matera, Phys. Rev. C 64, 045203 (2001).

[45] G. F. Bertsch, H. Kruse, and S. Das Gupta, Phys. Rev. C 29, 673 (1984).

[46] D. H. Boal, C. K. Gelbke, and B. K. Jennings, Rev. Mod. Phys. 62, 553 (1990).

[47] W. Bauer, C. K. Gelbke, and S. Pratt, Annu. Rev. Nucl. Part. Sci. 42, 77 (1992).

[48] D. Ardouin, Int. J. Mod. Phys. E 6, 391 (1997).

[49] U. A. Wiedemann and U. Heinz, Phys. Rep. 319, 145 (1999).

[50] W. G. Gong et al., Phys. Rev. Lett. 65, 2114 (1990).

[51] W. G. Gong, W. Bauer, C. K. Gelbke, and S. Pratt, Phys. Rev. C 43, 781 (1991).

[52] W. G. Gong et al., Phys. Rev. C 47, R429 (1993).

[53] G. J. Kunde et al., Phys. Rev. Lett. 70, 2545 (1993).

[54] D. O. Handzy et al., Phys. Rev. Lett. 75, 2916 (1995).

[55] G. Verde, D. A. Brown, P. Danielewicz, C. K. Gelbke, W. G. Lynch, and M. B. Tsang, Phys. Rev. C 65, 054609 (2002).

[56] G. Verde, P. Danielewicz, D. A. Brown, W. G. Lynch, C. K. Gelbke, and M. B. Tsang, Phys. Rev. C 67, 034606 (2003).

[57] R. Ghetti et al., Nucl. Phys. A674, 277 (2000).

[58] R. Ghetti et al., Phys. Rev. Lett. 87, 102701 (2001).

[59] R. Ghetti et al., Phys. Rev. Lett. 91, 092701 (2003).

[60] R. Ghetti et al., Phys. Rev. C 69, 031605 (2004).

[61] S. E. Koonin, Phys. Lett. 70B, 43 (1977).

[62] S. Pratt, Phys. Rev. Lett. 53, 1219 (1984); Phys. Rev. D 33, 72 (1986).

[63] S. Pratt and M. B. Tsang, Phys. Rev. C 36, 2390 (1987).

[64] S. Pratt, Nucl. Phys. A566, 103c (1994).

[65] P. E. Hodgson and E. Bĕták, Phys. Rep. 374, 1 (2003); and the references therein. 
[66] L. P. Csernai and J. I. Kapusta, Phys. Rep. 131, 223 (1986); and the references therein.

[67] M. Gyulassy, K. Frankel, and E. A. Relmer, Nucl. Phys. A402, 596 (1983).

[68] V. Koch et al., Phys. Lett. B 241, 174 (1990).

[69] P. Pawlowski et al., Eur. Phys. J. A 9, 371 (2000).

[70] R. Mattiello et al., Phys. Rev. Lett. 74, 2180 (1995); R. Mattiello et al., Phys. Rev. C 55, 1443 (1997).
[71] J. L. Nagle et al., Phys. Rev. C 53, 367 (1996).

[72] A. Polleri et al., Nucl. Phys. A661, 452c (1999).

[73] K. Hagel et al., Phys. Rev. C 62, 034607 (2000).

[74] J. Cibor et al., Phys. Lett. B 473, 29 (2000).

[75] L. G. Sobotka, R. J. Charity, and J. F. Dempsey, in [3], p. 331.

[76] M. Veselsky et al., Phys. Lett. B 497, 1 (2001).

[77] Ph. Chomaz and F. Gulminelli, Phys. Lett. B 447, 221 (1999). 